\title{
Optical and X-ray outbursts of Be/X-ray binary system SAX J2103.5+4545
}

\author{
Ü. Kızıloğlu, S. Özbilgen, N. Kızıloğlu, and A. Baykal
}

Physics Department, Middle East Technical University, Ankara 06531, Turkey

e-mail: umk@astroa.physics.metu.edu.tr

Received 5 June 2009 / Accepted 13 October 2009

\section{ABSTRACT}

\begin{abstract}
Aims. The main goal of this study is to investigate the relationship between the optical and X-ray behaviours of the Be/X-ray binary system SAX J2103.5+4545.

Methods. We present the relations between the $\mathrm{H} \alpha$ equivalent width, optical brightness and X-ray flux of the system, by analysing the optical photometric and spectroscopic observations together with the X-ray observations .

Results. The X-ray outburst of the system occurred just after the optical outburst. The nearly symmetric $\mathrm{H} \alpha$ emission line profiles observed during the beginning of the optical outburst turn into asymmetric profiles with increased EW values during the dissipation of the Be disc. A correlation between the $\mathrm{H} \alpha$ emission line strength and the X-ray flux was found. The $\mathrm{H} \alpha$ line indicates the existence of an equatorial disc around the $\mathrm{Be}$ star. The $\mathrm{H} \alpha$ line changed from emission to absorption during the observation period. The observed double peaked $\mathrm{HeI}$ emission lines might come from the accretion disc of neutron star which is temporarily formed at the time of X-ray outburst.
\end{abstract}

Key words. stars: emission-line, $\mathrm{Be}$ - stars: early-type - stars: variables: general - X-rays: binaries

\section{Introduction}

\subsection{Be/X-ray systems}

The Be/X-ray systems consist of a Be star and a neutron star. Be stars are close to the main-sequence. They exhibit Balmer lines in emission and show strong infrared excess (compared to normal stars of the same stellar spectral type) in their spectra. The disappearance and appearance of emission lines suggest a disc structure around the Be star (Slettebak 1988; Okazaki \& Negueruela 2001). The disc formation is related to the material lost from the rapidly rotating Be star (Porter \& Rivinius 2003). The motions of discs are rotationally dominated and quasi Keplerian (Hanuschik 1996). The interaction of neutron star with the Be disc (Stella et al. 1986; Negueruela et al. 1998) causes $\mathrm{X}$-ray outbursts either at the periastron passage (type I outburst with X-ray luminosity $\sim 10^{36}-10^{37} \mathrm{erg} / \mathrm{s}$ ) or at any orbital phase (type II outburst with X-ray luminosity greater than $10^{37} \mathrm{erg} / \mathrm{s}$ ). There is also X-ray quiescence phase where the accretion onto the NS is partially or completely stopped.

In some Be/X-ray systems the Be disc forms, grows, causes an X-ray outburst and disappears (Negueruela et al. 2001; Reig et al. 2001; Reig et al. 2005b; Baykal et al. 2005; Reig et al. 2007). Some systems display an X-ray outburst and do not go to a disc loss phase (Wilson et al. 2002, 2005; Kizıloğlu et al. 2007; Baykal et al. 2008). It is also possible to observe long periods of $\mathrm{X}$-ray quiscence even when a Be disc is present (Kızıloğlu et al. 2007).

The favoured disc model is the viscous disc model (Okazaki 2001; Okazaki \& Negueruela 2001). Okazaki \& Negueruela (2001) applied their resonantly truncated viscous disc model to some Be/X-ray systems. They found that systems with high orbital eccentricity show regular type I outbursts while systems with low orbital eccentricity display occasional giant type II outbursts. Be/X-ray systems with moderate eccentricity and relatively close orbits have efficient disc truncation which allows the material to be stored in the Be disc. When the disc radius is larger than the Roche lobe radius, type I outbursts result. If the Be disc is smaller than the critical lobe radius, type II outbursts are displayed and temporary type I outbursts are seen when the Be disc is strongly disturbed. If the Be disc is elongated toward the periastron then material is transferred around the periastron forming an accretion disc around the NS.

\section{2. $S A X J 2103.5+4545$}

The transient X-ray source SAX J2103.5+4545 was discovered on February 1997 by the BeppoSAX Wide Field Camera (Hulleman et al. 1998). The source was active between February and September 1997, with a pulse period of 358.61 s. The next activity was two years later on November 1999 and was detected by the all-sky monitor (ASM) on board of RXTE. Using X-ray data from RXTE, Baykal et al. (2000) determined the orbital parameters of the system by Doppler shift analysis of the pulsations. These observations indicated a high mass companion in the system. SAX J2103.5+4545 had another X-ray activity in July 2002. Orbital solutions for SAX J2103.5+4545 showed that this system had a moderately eccentric orbit $(e=0.4)$ with an orbital period of $12.66 \mathrm{~d}$ (Baykal et al. 2007). Baykal et al. (2007) obtained a distance of $4.5 \pm 0.5 \mathrm{kpc}$ to this source. They reported a correlation between spin-up/down trend and an X-ray flux which could be explained by accretion from an accretion disc onto the NS.

Reig et al. (2004) identified a highly reddened optical counterpart $(V=14.2 \mathrm{mag})$ to SAX J2103.5+4545. They suggested a spectral type of B0Ve for the optical companion whose distance was suggested to be $6.5 \pm 0.9 \mathrm{kpc}$. During their 
spectroscopic observations, a double peak $\mathrm{H}_{\alpha}$ emission line was observed which was an indication of a disc surrounding Be star. Less than a month later the $\mathrm{H}_{\alpha}$ line was seen in absorption (after MJD 52870). Blay et al. (2006) presented the evolution of $\mathrm{H}_{\alpha}$ line profiles between August 2003 and September 2004. They noticed that the transition of the $\mathrm{H}_{\alpha}$ line from emission to absorption occurs in coincidence with the transition of the $\mathrm{Be} / \mathrm{X}$-ray system from a higher to lower X-ray activity state. Although Be star lost its disc after the August 2003, the X-ray emission was detected due to the accretion of material on to the NS. Reig et al. (2005a) concluded that the X-ray emission with a luminosity of $3-5 \times 10^{35} \mathrm{erg} / \mathrm{s}$ during the quiescent phase must originate in the stellar wind. The system was in a low activity state since the end of 2003.

The first detection of the $\mathrm{H}_{\alpha}$ emission since August 2003 was made by Manousakis et al. (2007) on MJD 54234 and 54240 where they observed double peaked emission lines with equivalent widths of 2.5 and $1.9 \AA$, respectively, after the report of X-ray outburst from SAX J2103.5+4545 (Galis et al. 2007; Krimm et al. 2007). Galis et al. (2007) reported a hard X-ray outburst on April 27, 2007 (MJD 54218) which was detected by INTEGRAL IBIS/ISGRI with a flux of $1.7 \pm 0.1 \times 10^{-9} \mathrm{erg} / \mathrm{cm}^{2} / \mathrm{s}$ and $5.3 \pm 0.7 \times 10^{-10} \mathrm{erg} / \mathrm{cm}^{2} / \mathrm{s}$ in the $20-40 \mathrm{keV}$ and $40-80 \mathrm{keV}$ energy band, respectively.

In this study, we present the relationship between the longterm X-ray and the optical variability using observations from both RXTE/ASM and ROTSEIIId as well as the spectral analysis of the Be/X-ray system SAX J2103.5+4545. We discuss the correlated behaviours in the optical and X-ray wavelengths. The behaviour of the optical variation of the Be star helps to understand the X-ray behaviour of the system. We discuss the evidences in both the $\mathrm{H}_{\alpha}$ and HeI profiles.

\section{Observations and data reduction}

The optical data were obtained with Robotic Optical Transient Experiment $^{1}$ (ROTSEIIId) and the Russian-Turkish $1.5 \mathrm{~m}$ Telescope $^{2}$ (RTT150) located at Bakırlitepe, Antalya, Turkey.

\subsection{Optical photometric observations}

The ROTSEIIId telescope has an aperture of $45 \mathrm{~cm}$. It is equipped with a $2048 \times 2048$ pixel CCD and has a field of view of $1.85 \times 1.85$. Its pixel scale is $3.3^{\prime \prime} /$ pixel. The system operates without filters and has a wide pass-band which peaks at $5500 \AA$ (Akerlof et al. 2003).

The photometric observations continue since June 2004 (MJD 53167) with a break in observations between August 2005 and April 2007. Approximately $5000 \mathrm{CCD}$ frames with a $5 \mathrm{~s}$ exposure time were obtained. All images were automatically dark and flat field corrected by a data extraction pipeline of ROTSEIIId (Akerlof et al. 2003), which feeds corrected frames to the Sextractor package (Bertin \& Arnouts 1996). Instrumental magnitudes were obtained using Sextractor aperture photometry on the observed CCD frames. ROTSE magnitudes were calibrated by comparing all the field stars against the USNO A2.0 $R$-band catalog. Apart from the data reduction pipeline, all CCD images were checked for image quality, and PSF photometry was applied using MIDAS and its DAOPHOT package (Stetson 1987, 1992). Barycentric corrections were applied to

\footnotetext{
1 http://www . rotse.net

2 http://www . tug.tubitak.gov.tr
}
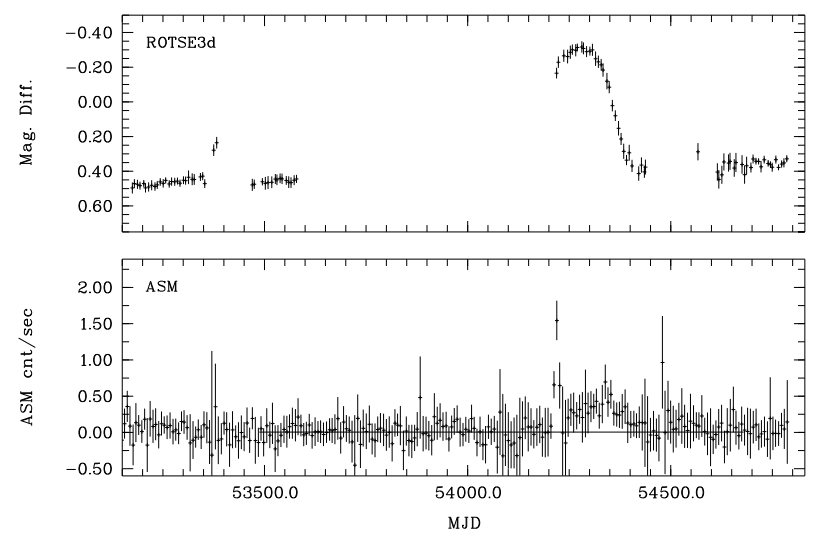

Fig. 1. Weekly averages of ROTSEIIId differential light curve of the Be star (top panel) and the X-ray light curve obtained from RXTE/ASM data (bottom panel).

Table 1. Optical counterpart to SAX J2103.5+4545 and photometric reference stars.

\begin{tabular}{cccc}
\hline \hline Star & RA & Dec & USNO A2 \\
& $(\mathrm{J} 2000.0)$ & $(\mathrm{J} 2000.0)$ & $R$ mag \\
\hline Optical counterpart to & & & \\
SAX J2103.5+4545 & $21^{\mathrm{h}} 03^{\mathrm{m}} 35^{\mathrm{s} .7}$ & $+45^{\circ} 45^{\prime} 04^{\prime \prime} 0$ & 14.4 \\
Star 1 & $21^{\mathrm{h}} 03^{\mathrm{m}} 20^{\mathrm{s}} .15$ & $+45^{\circ} 45^{\prime} 14^{\prime \prime} 6$ & 12.1 \\
Star 2 & $21^{\mathrm{h}} 03^{\mathrm{m}} 21^{\mathrm{s}} .99$ & $+45^{\circ} 44^{\prime} 55^{\prime \prime} 5$ & 13.8 \\
Star 3 & $21^{\mathrm{h}} 03^{\mathrm{m}} 40^{\mathrm{s}} .32$ & $+45^{\circ} 46^{\prime} 22^{\prime \prime} 6$ & 13.0 \\
\hline
\end{tabular}

the times of each observation by using JPL DE200 ephemerides. Details on the reduction of data were described in Kızıloğlu et al. (2005).

Three reference stars were determined by examining the system's neighbourhood for stars relatively constant magnitudes (Table 1). The average magnitude for reference stars was calculated after the magnitudes and magnitude errors for these stars were obtained from the appropriate frames. To obtain the differential magnitude, the average of the reference star's magnitudes is subtracted from the Be star's magnitude (Fig. 1 top panel).

\subsection{Optical spectroscopic observations}

RTT150 was used for spectroscopic observations with the medium resolution spectrometer TFOSC (TÜBİTAK Faint Object Spectrometer and Camera) on the focal plane of the telescope. The camera has a $2048 \times 2048,15 \mu$ pixel Fairchild 447BI CCD whose fov is $13 \times 13$ arcmin with a pixel scale of $0.39^{\prime \prime} /$ pixel. We used grism G8 (5800-8300 $\AA$ ) with average dispersion $\sim 1.1 \AA$ /pixel. Eight spectroscopic observations have been made with TFOSC starting in June 2007 until September 2008. A journal of optical spectroscopic observations of SAX J2103.5+4545 is given in Table 2. The spectra were reduced by using MIDAS $^{3}$ and its packages: Longslit context and ALICE.

\subsection{RXTE/ASM and SWIFT/BAT observations}

The observations of the All Sky Monitor (ASM) on board the Rossi X-ray Timing Explorer (RXTE) satellite (Levine et al. 1996) and the Burst Alert Telescope $(B A T)$ on board of the

\footnotetext{
${ }^{3}$ http://www.eso.org/projects/esomidas/
} 
Table 2. Journal of spectroscopic observations for the $\mathrm{H} \alpha$ and $\mathrm{HeI}$ lines.

\begin{tabular}{lcccc}
\hline \hline Date & $\begin{array}{c}\mathrm{MJD} \\
(\AA)\end{array}$ & $\begin{array}{c}\mathrm{EW}^{a}(\mathrm{H} \alpha) \\
(\AA)\end{array}$ & $\begin{array}{c}\mathrm{EW}(\mathrm{HeI}) \\
6678 \AA\end{array}$ & $\begin{array}{c}\text { EW (HeI) } \\
7065 \AA\end{array}$ \\
\hline 2007 Jun. 14 & 54265.9 & $1.03 \pm 0.12$ & $-0.32 \pm 0.11$ & $-0.23 \pm 0.11$ \\
2007 Jul. 18 & 54299.9 & $1.66 \pm 0.15$ & $-0.49 \pm 0.10$ & $-0.12 \pm 0.09$ \\
2007 Sep. 13 & 54356.9 & $5.20 \pm 0.14$ & $0.56 \pm 0.11$ & $0.67 \pm 0.12$ \\
2007 Oct. 05 & 54378.8 & $3.22 \pm 0.13$ & $0.59 \pm 0.14$ & $0.65 \pm 0.09$ \\
2007 Dec. 14 & 54448.8 & $-2.46 \pm 0.19$ & $-0.75 \pm 0.36$ & $-0.69 \pm 0.15$ \\
2008 Jun. 19 & 54636.9 & $-2.54 \pm 0.18$ & $-0.61 \pm 0.16$ & $-0.64 \pm 0.27$ \\
2008 Aug. 26 & 54704.9 & $-2.32 \pm 0.18$ & $-0.93 \pm 0.32$ & $-0.71 \pm 0.16$ \\
2008 Sep. 22 & 54731.0 & $-2.44 \pm 0.16$ & $-0.75 \pm 0.27$ & $-0.84 \pm 0.18$ \\
\hline
\end{tabular}

${ }^{a}$ Positive values stand for emission line EWs whereas absorption EWs are denoted as negative.

$S W I F T^{4}$ satellite were used to compare the optical results with the X-ray observations. The ASM energy range is from 1.3 to $12 \mathrm{keV}$. In this study we used the $5-12 \mathrm{keV}$ band observations. The BAT provides observations in the energy range $15-50 \mathrm{keV}$. The light curves of several targets are available in the public archive of SWIFT/BAT.

\section{Results and discussion}

\subsection{Optical and $X$-ray variability}

The ROTSEIIId light curve (weekly averages) of the Be/X-ray binary system SAX J2103.5+4545 was shown in Fig. 1 together with the ASM X-ray light curve in the 5-12 keV energy band. The precision of photometry is around 0.03 mag for the individual observation. The optical light curve shows a very slow increase in its brightness during the earlier period of observations. An optical outburst was observed around the time MJD 54200. Such behaviour has previously been seen in only a few Be/X-ray systems such as 4U 0115+635 (Negueruela et al. 2001; Baykal et al. 2005; Reig et al. 2007), V 0332+53 (Negueruela et al. 1999; Goranskij 2001; Kızıloğlu et al. 2008) and A 0535+26 (Clark et al. 1999; Negueruela et al. 2000; Coe et al. 2006). The brightening of the system is of about a magnitude of 0.7 . We infer from such a brightening that a disc is formed in the equatorial plane surrounding the Be star. The Be disc is assumed to be fed from the material that is lost from the Be star. Figure 2 shows the plot of daily averages of the observations during the optical and X-ray outbursts. The same figure also shows the $\mathrm{H} \alpha$ equivalent width (EW) values. During the optical outburst of the system ASM observations show increase in the count rates. BAT observations also show a similar picture. The X-ray brightening lasts more than one orbital period.

According to the model of Okazaki \& Negueruela (2001) the present system brightens in optical wavelenghts until the Be disc is truncated by the NS. X-ray outburst occurred at MJD 54213 (about 3 days after the periastron passage, PAP), reaching its maximum value at MJD 54218 (Krimm et al. 2007) just before the Be star reaches its maximum brightness. The X-ray activity continues after the X-ray outburst and periodic small amplitude outbursts are seen superimposed on an increased X-ray emission after MJD 54250. The average X-ray luminosity increases till MJD 54350 and then decreases with the brightness of the Be star. Considering the X-ray flux $\left(1.7 \pm 0.1 \times 10^{-9} \mathrm{erg} / \mathrm{cm}^{2} / \mathrm{s}\right)$ detected by INTEGRAL (Galis et al. 2007), the luminosity of the source was calculated as $\sim 4 \times 10^{36}$ and $\sim 8.5 \times 10^{36} \mathrm{erg} / \mathrm{s}$ for distances

${ }^{4}$ http://swift.gsfc.nasa.gov/docs/swift/results/
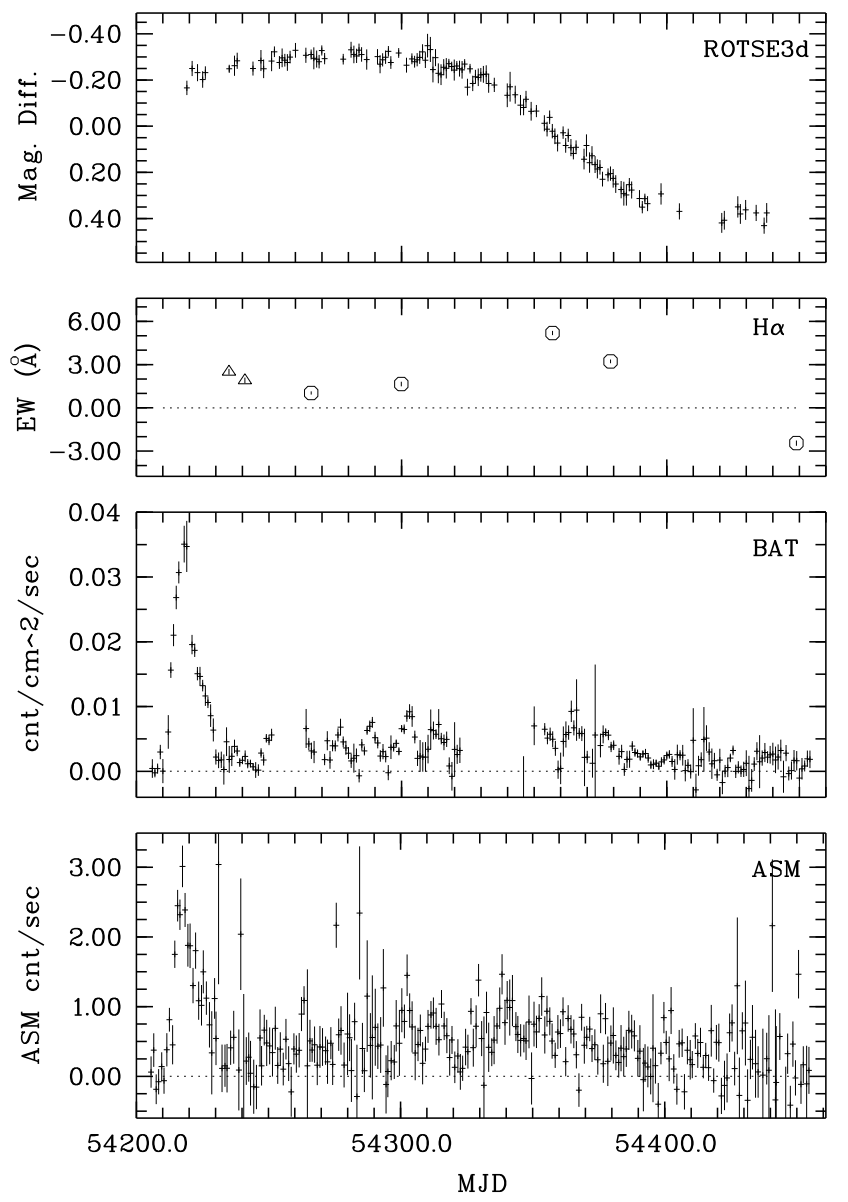

Fig. 2. Post-outburst daily averages of the differential light curve of the $\mathrm{Be}$ star (top panel) and the $\mathrm{H} \alpha$ equivalent widths (second panel). Triangles denote values taken from Manousakis et al. (2007). The two bottom panels show daily averages of SWIFT/BAT 15-50 keV and RXTE/ASM 5-12 keV X-ray light curves.

$4.5 \mathrm{kpc}$ (Baykal et al. 2007) and $6.5 \mathrm{kpc}$ (Reig et al. 2004), respectively. These luminosities are similar to the previously measured X-ray outburst values (Baykal et al. 2007; Camero Arranz et al. 2007). Although the luminosity is hardly $10^{37} \mathrm{erg} / \mathrm{s}$, this outburst may be called a type II outburst (Camero Arranz et al. 2007). As seen in the ASM and BAT observations (Fig. 2), the duration of the outburst is more than one orbital period. After the type II outburst is completed, type I outbursts are observed at each periastron passage of the NS. This X-ray activity lasts about 6 months till the Be disc disappears completely. Considering a mean X-ray luminosity of $\sim 6 \times 10^{36} \mathrm{erg} / \mathrm{s}$ the mass accretion rate is found to be $\sim 3 \times 10^{16} \mathrm{gr} / \mathrm{s}$. This implies a mass transfer of $\sim 3 \times 10^{-11} M_{\odot}$ in 20 days during the X-ray outburst which is a small fraction of the total mass of the Be disc (Reig et al. 2007).

We folded the optical and X-ray observations at the period of $12.665 \mathrm{~d}$ using the epoch of the periastron passage time ( $T=$ MJD $52550.736+12.665 \mathrm{~N}$ ) in order to see whether there is any orbital period signature in the optical and X-ray light curve or not. A polynomial was fitted to the optical light curve to remove the long term variation, and the residual time series were folded with the expected period. The folded optical and SWIFT/BAT light curves for the observations after MJD 54200 are shown in Fig. 3. A similar curve obtained from the SWIFT/BAT observations prior to the X-ray outburst is also shown in the same figure. There is no modulation of the optical light curve at the orbital period (with a statistical significance $\chi_{v}^{2}=3.1$ ). However, the 


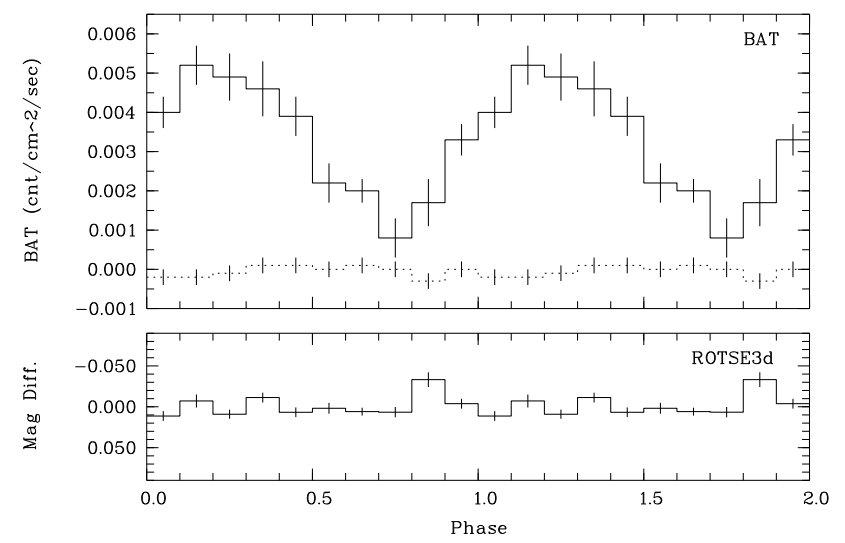

Fig. 3. Top panel: the solid line shows SWIFT/BAT $15-50 \mathrm{KeV}$ post-outburst observations folded at the period $12.665 \mathrm{~d}$ using the ephemerides $(T=$ MJD $52550.736+12.665 \mathrm{~N})$. The dotted line shows the pre-outburst observations. Bottom panel: folded optical light curve at the same orbital period after long term variation is removed.

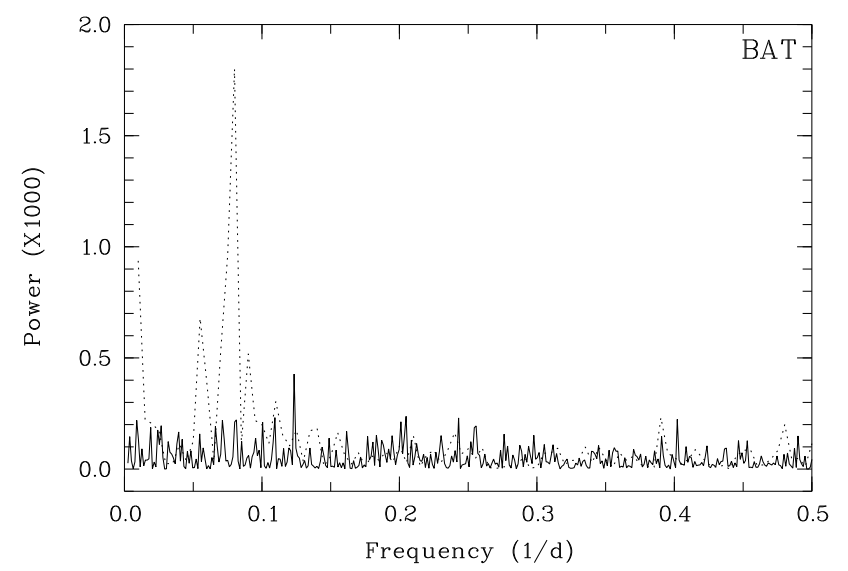

Fig. 4. Power spectra of SAX J2103.5+4545 obtained from the 15$50 \mathrm{keV}$ SWIFT/BAT pre-outburst (solid line) and post-outburst (dotted line) observations.

orbital period is detected with a $\chi_{v}^{2}=12.5$ for X-ray observations obtained after the X-ray outburst. The orbital modulation in the X-ray observations shows the existence of type I outbursts which occur periodically at each PAP of NS. Pre-outburst time series does not show any modulation. Figure 4 shows the power spectrum of BAT observations obtained by using the Scargle (1982) method. The calculated frequency for post X-ray outburst observations is $0.079 \mathrm{~d}^{-1}(12.6 \mathrm{~d})$, which is the orbital frequency (period) of the system. The detection is above $5 \sigma$ confidence level. We also searched for short-term variabilities in the optical light curve over a frequency range from 0 to $20 \mathrm{~d}^{-1}$. No variability is observed on shorter time scales.

The time for the type I outburst peaks were estimated to see the orbital phasing of outbursts. We fitted a Gaussian model to the type I outbursts obtained from the daily averaged light curves of SWIFT/BAT (15-50 keV) and RXTE/ASM (5-12 keV) observations. We centered the Gaussian model on the approximated type I outburst profiles. In the fitting, the center value, the amplitude and the width of Gaussian were taken as free parameters. We then found the peak time of the outburst. A similar application has been done previously for the Be/X-ray system EXO 2030+375 (Baykal et al. 2008). In Fig. 5, we present the calculated peak time of the observed outburst minus the calculated time of the periastron passage at the time of the

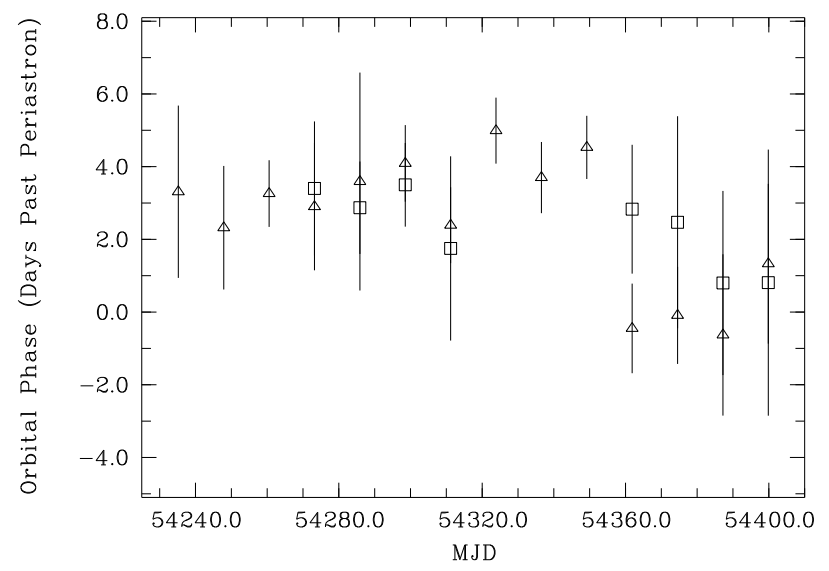

Fig. 5. Orbital phase of SAX J2103.5+4545 type I outburst peaks versus time. The open squares and open triangles indicate the orbital phases estimated from the $S W I F T /$ BAT $15-50 \mathrm{keV}$ light curve and RXTE/ASM 5-12 keV light curve, respectively.

outburst versus time. The times of outburst peaks estimated from Gaussian fits to both the RXTE/ASM and SWIFT/BAT observations vary about 3 days after PAP. Baykal et al. (2000) and İnam et al. (2004) found a similar time delay after PAP when the X-ray flux was maximum. A slowly precessing density wave in the Be disc may induce type I outbursts at the time 3 days after the PAP time, when the Be disc is elongated toward the periastron. The larger differences after MJD 54350, during the dispersion of the Be disc, between the outburst peak times for ASM and BAT observations are probably due to the difficulty in determining the peak positions of the outbursts. The X-ray outburst luminosity decreases as the Be disc disappears, and the uncertainities in the lower energy RXTE observations are larger compared to those of BAT.

\subsection{Analysis of the $\mathrm{H} \alpha$ profiles}

The observed $\mathrm{H} \alpha$ profiles and their EW values are given in Fig. 6 and Table 2, respectively. The first two double peaked emission profiles have a similar structure and EWs as those given by Reig et al. (2004). The EW values of the first two profiles are also similar to those of Manousakis et al. (2007) who made observations about a month after the X-ray outburst. The other two emission profiles have a more complex structure. Only a blue peak is visible. In Fig. 2 we also plot the EW values of the $\mathrm{H} \alpha$ emission line given by Manousakis et al. (2007) in addition to our values. The system shows weak $\mathrm{H} \alpha$ emission at the time of the maximum optical brightening of the system. The EWs observed about four months after the X-ray outburst are larger than its previous values. There seems to be no correlation between the $\mathrm{H} \alpha$ emission EWs and the optical brightness of the system. After MJD 54400 the Be disc disappears, and the $\mathrm{H} \alpha$ lines are observed in absorption. Such disc formation and disc loss phases are seen in A0535+26 (Clark et al. 1999; Negueruela et al. 2000) and 4U 0115+63 (Negueruela et al. 2001; Baykal et al. 2005; Reig et al. 2007).

Negueruela et al. (1998) noted that the $\mathrm{H} \alpha$ emission region was affected during the type II outburst by the perturbation in the Be disc which occurred immediately after the type II outburst. When the density perturbations interact with the NS type I outbursts occur. They presented observational evidence for large density perturbations in the Be discs of Be/X-ray systems like 4U 0115+634, V0332+53 and A0535+262, which 


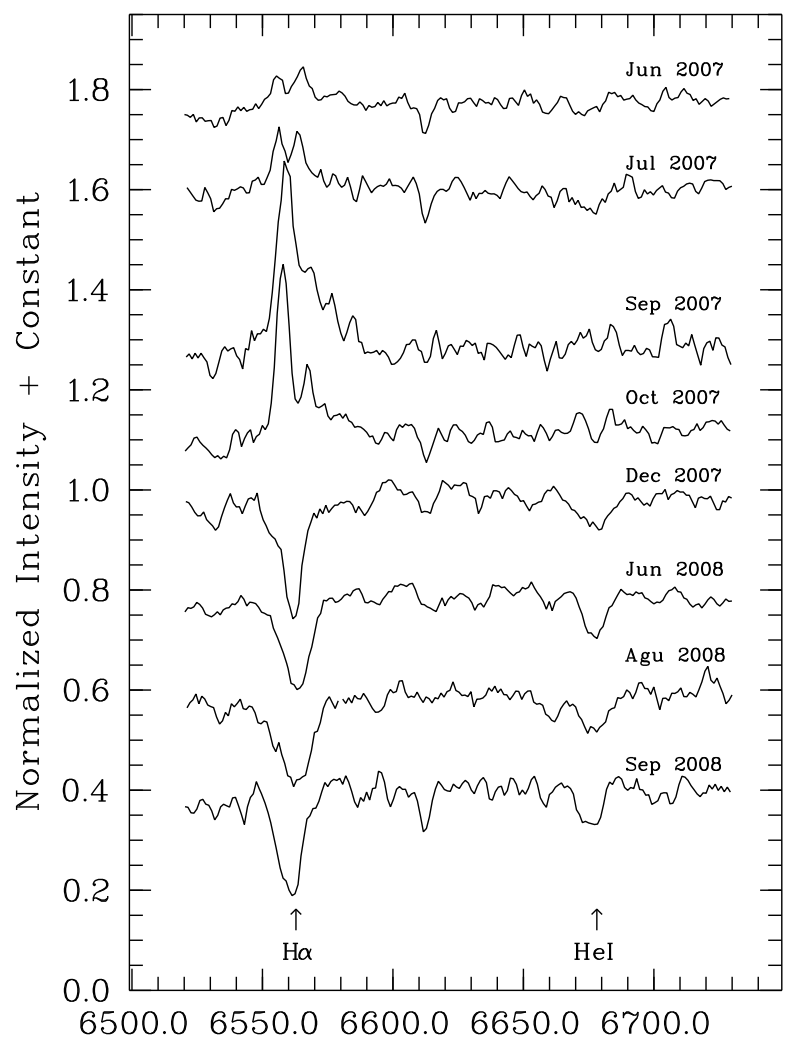

Wavelength $(\AA)$

Fig. 6. $\mathrm{H} \alpha$ and HeI ( $\lambda$ 6678) profiles observed after The type II outburst. Each profile normalised and properly offsetted.

are moderately eccentric systems. Asymmetric shapes of the $\mathrm{H} \alpha$ line profiles are due to density perturbation which develops $\mathrm{V} / \mathrm{R}$ variability. The variations in the shape of the $\mathrm{H} \alpha$ emission profiles (V/R variability) show a global change in the structure of the Be disc. Negueruela et al. (1998) explained the observed $\mathrm{V} / \mathrm{R}$ variations with the models of one-armed oscillations. They found a correlation between type II outburst and density perturbation. Reig et al. (2005b) suggested that the effect of density perturbation was not seen in the shape of the $\mathrm{H} \alpha$ lines until the disc was fully formed, that is after the disc size and density reached a critical value. In the present study the $\mathrm{H} \alpha$ profiles do not reflect a perturbation just after the X-ray outburst. They show a quasi symmetric form. At the time of the X-ray outburst it is not clearly understood whether the Be disc is distorted or not. When the system reaches its maximum brightness and the Be disc is fully developed the EWs of these profiles are less than $\sim 2 \AA$. The first asymmetric profile is seen when EW of the $\mathrm{H} \alpha$ line is $\sim 5 \AA$. We observe asymmetric $\mathrm{H} \alpha$ line profiles with sharp blue peak and larger EW values during the decay of the Be disc. This indicates a structural change in the outer layers of the Be disc after MJD 54300. If a density perturbation is responsible for the complex profile of the $\mathrm{H} \alpha$ it is observed during the dissipation of the Be disc when the size or density of the disc decreases.

As Reig et al. (2007) explained, the surface density of the $\mathrm{Be}$ disc increases rapidly in Be/X-ray binaries due to the truncation of the disc. The disc becomes optically thick at IR wavelengths, and becoming instable, it begins to warp, tilt and precess. The high values of the $\mathrm{H} \alpha \mathrm{EW}$ in $4 \mathrm{U} 0115+63$ happen because of a warped disc which presents a larger surface area to the observer. In the present system SAX J2103.5+4545, the
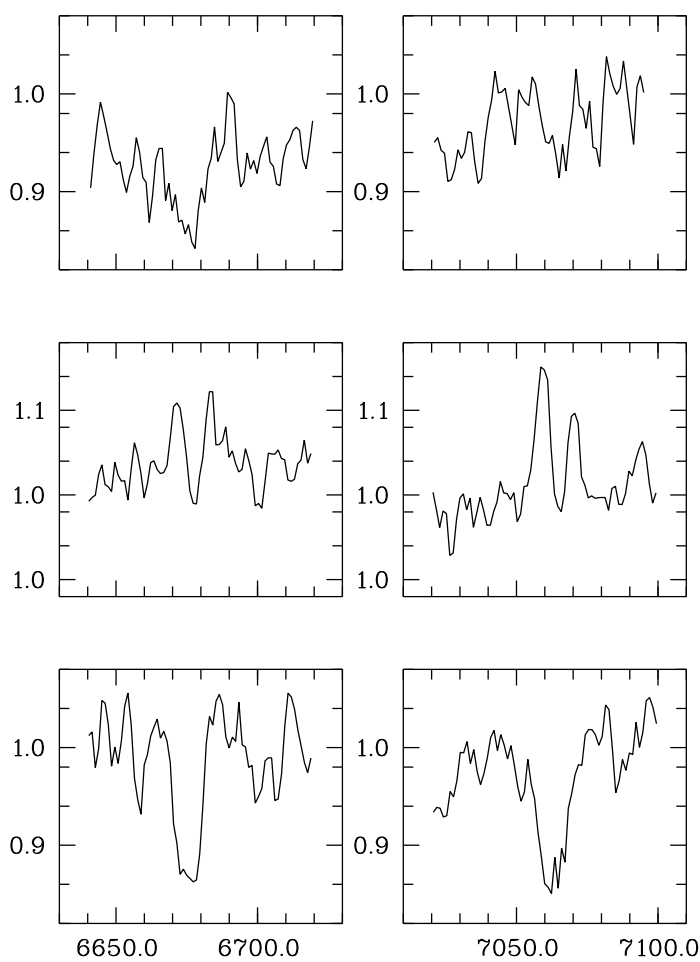

Fig. 7. HeI $\lambda \lambda 6678,7065 \AA$ profiles observed after the X-ray outburst. Top panel: on MJD 54299.9. Mid panel: on MJD 54378.8. Bottom panel: on MJD 54731.0.

increase in the $\mathrm{H} \alpha \mathrm{EW}$ and the distortion in the structure of the blue peaked $\mathrm{H} \alpha$ profile during the decrease of the optical brightening can be seen as a result of the warping of the disc. An instability in the Be disc causes the outer layers to warp, showing a larger surface area to the observer.

\subsection{The Hel $\lambda \lambda 6678,7065 \AA$ lines}

The EWs of the HeI $\lambda \lambda 6678,7065 \AA$ lines are given in Table 2. Both lines behave similarly as seen in Fig. 7. The HeI lines show a double peak emission feature in the two spectra on MJD 54356 and 54378. The double peaked HeI features are typical in the early Be stars without compact companions (Dachs et al. 1992; Negueruela et al. 2004). These emission lines were also observed in the Be/X-ray systems like RX J0440.9+4431 (Reig et al. 2005b) and A0535+26 (Clark et al. 1998; Coe et al. 2006). In the first two spectra obtained on MJD 54265 and 54299 the HeI lines are not seen well. However a weak absorption feature can be deduced. During these observations the optical brightness is at its highest value. The HeI feature is seen in absorption in the remaining series of the spectra where the optical brightness has returned to its pre-outburst value.

The relation between the $\mathrm{H} \alpha$ emission strength and the X-ray flux (Fig. 2) shows that the increase in the X-ray luminosity is accompanied by an increase in the EWs of the $\mathrm{H} \alpha$ lines. Higher EW values of the $\mathrm{H} \alpha$ emission line and the HeI double peak emission lines occur during the decay of the Be disc. It might be possible that a temporary accretion disc formed around a neutron star at the time of the X-ray outburst contributes to the emission of the $\mathrm{H} \alpha$ and HeI lines. Giovanelli et al. (2007) argued that the double peak HeI emission lines observed in the Be/X-ray binary system A0535+26 was formed in the temporary accretion disc of NS. They calculated the radius for the HeI $6678 \AA$ emission line and found that this radius was incompatible with the 
stellar radius. They decided that the doubling of the HeI emission lines was due to the accretion disc. The presence of a temporary accretion disc for SAX J2103.5+4545 may be questionable. We are not able to show the existence of such a temporary disc during the last X-ray outburst. However, Baykal et al. (2007) obtained a correlation between spin up/down and X-ray flux for SAX J2103.5+4545 during its previous two X-ray outbursts and explained this by accretion from an accretion disc. Very recently, Reig et al. (2009) found a correlation between the strength and shape of the $\mathrm{H} \alpha$ line originated from the Be star and the X-ray emission from the neutron star.

\section{Summary}

Be/X-ray binary system SAX J2103.5+4545 has been monitored since June 2004 with a break in observations between August 2005 and April 2007. The system shows four X-ray outbursts during the last ten years. The $2007 \mathrm{X}$-ray outburst coincides with the optical outburst of the system. The optical brightening of the system of about the magnitude of 0.7 . The $\mathrm{H} \alpha$ emission lines observed during this time show the existence of a disc surrounding the Be star. The $\mathrm{H} \alpha$ emission profiles go from symmetric profiles to asymmetric ones in about four months. The appearance of HeI double peak emission lines occurred when there is a structural change in the $\mathrm{H} \alpha$ profile. Both lines ( $\mathrm{H} \alpha$ and $\mathrm{HeI}$ lines) show an increase in EW values during the decay of the Be disc. The peak times of the type I outbursts are about three days after the PAP of the neutron star. Temporary type I outbursts continues about 12 orbits after the X-ray outburst. After the decay of the Be disc the emission lines of the $\mathrm{H} \alpha$ and HeI turn into absorption lines and the system is still in optic quiscence.

Acknowledgements. We thank the anonymous referee for a careful reading and valuable comments. This project utilizes data obtained by the Robotic Optical Transient Search Experiment. ROTSE is a collaboration of Lawrence Livermore National Lab, Los Alamos National Lab, and the University of Michigan (http://www.rotse.net). We thank the Turkish National Observatory of TÜBİTAK for running the optical facilities. We acknowledge support from TÜBİTAK, The Scientific and Technological Research Council of Turkey, through project $106 \mathrm{~T} 040$. We also acknowledge the $R X T E / \mathrm{ASM}$ team for the $\mathrm{X}$-ray monitoring data. SWIFT/BAT transient monitor results were provided by the $S W I F T /$ BAT team.

\section{References}

Akerlof, C. W., Kehoe, R. L., McKay, T. A., et al. 2003, PASP, 115, 132 Baykal, A., Stark, M. J., \& Swank, J. H. 2000, ApJ, 544, L129

Baykal, A., Kiziloglu, U., Kiziloglu, N., et al. 2005, A\&A, 439, 1131 Baykal, A., İnam, S. C., Stark, M. J., et al. 2007, MNRAS, 374, 1108 Baykal, A., Kiziloglu, U., Kiziloglu, N., et al. 2008, A\&A, 479, 301 Bertin, E., \& Arnouts, S. 1996, A\&AS, 117, 393

Blay, P., Camero, A., Martinez-Nunez, S., et al. 2006, ESA SP-604, 243 Camero Arranz, A., Wilson, C. A., Finger, M. H., \& Reglero, V. 2007, A\&A, 473,551

Clark, J. S., Tarasov, A. E., Steele, I. A., et al. 1998, MNRAS, 294, 165

Clark, J. S., Lyuty, V. M., Zaitseva, G. V., et al. 1999, MNRAS, 302, 167 Coe, M. J., Reig, P., McBride, V. A., et al. 2006, MNRAS, 368, 447 Dachs, J., Hummel, W., \& Hanuschik, R. W. 1992, A\&AS, 95, 437 Galis, R., Beckmann, V., Bianchin, V., et al. 2007, ATel \#1063 Giovannelli, F., Bernabei, S., Rossi, C., et al. 2007, A\&A, 475, 651 Goranskij, V. 2001, AstL, 27, 516

Hanuschik, R. W., Hummel, W., Sutorius, E., et al. 1996, A\&AS, 116, 309 Hulleman, F., in 't Zand, J. J. M., \& Heise, J. 1998, A\&A, 337, L25 İnam, S. C., Baykal, A., Swank, J. H., et al. 2004, ApJ, 616, 453 Kızıloğlu, U., Kızıloğlu, N., \& Baykal, A. 2005, AJ, 130, 2766 Kızıloğlu, U., Kızıloğlu, N., Baykal, A., et al. 2007, A\&A, 470, 1023 Kızıloğlu, U., Kızıloğlu, N., \& Baykal, A., et al. 2008, IBVS, 5865 Krimm, H. A., Barthelmy, S. D., Barbier, L., et al. 2007, ATel \#1064 Levine, A. M., Bradt, H., Cui, W., et al. 1996, ApJ, 496, L33

Manousakis, A., Reig, P., \& Kougentakis, A. 2007, ATel \#1085 Negueruela, I., Reig, P., Coe, M. J., et al. 1998, A\&A, 336, 251 Negueruela, I., Roche, P., Fabregat, J., et al. 1999, MNRAS, 307, 695 Negueruela, I., Reig, P., Finger, M. H., et al. 2000, A\&A, 356, 1003 Negueruela, I., Okazaki, A. T., Fabregat, J., et al. 2001, A\&A, 369, 117 Negueruela, I., Steele, I. A., \& Bernabeu, G. 2004, AN, 325, 749 Okazaki, A. T. 2001, PASJ, 53, 119

Okazaki, A. T., \& Negueruela, I. 2001, A\&A, 377, 161

Porter, J. M., \& Rivinius, T. 2003, PASP, 115, 1153

Reig, P., Negueruela, I., Buckley, D. A. H., et al. 2001, A\&A, 367, 266

Reig, P., Negueruela, I., Fabregat, J., et al. 2004, A\&A, 421, 673

Reig, P., Negueruela, I., Papamastorakis, G., et al. 2005a, A\&A, 440, 637

Reig, P., Negueruela, I., Fabregat, J., et al. 2005b, A\&A, 440, 1079

Reig, P., Larionov, V., Negueruela, I., et al. 2007, A\&A, 462, 1081

Reig, P., Slowikowska, A., Zezas, A., et al. 2009, MNRAS, in press [arXiv: 0908.4497]

Scargle, J. D. 1982, ApJ, 263, 835

Slettebak, A. 1988, PASP, 100, 770

Stella, L., White, N. E., \& Rosner, R. 1986, ApJ, 208, 669

Stetson, P. B. 1987, PASP, 99, 191

Stetson, P. B. 1992, ASP Conf. Series, 25, 297

Wilson, C. A., Finger, M. H., Coe, M. J., et al. 2002, ApJ, 570, 287

Wilson, C. A., Weisskopf, M. C., Finger, M. H., et al. 2005, ApJ, 622, 1024 